\title{
Theoretical Neuroscience: Modeling the Activation Mechanism of Potassium Channels in Neurons
}

\author{
Kevin Twedt \\ Department of Physics \\ University of Northern lowa \\ Cedar Falls, lowa 50614-0150 USA
}

Received: October 20, 2003 Accepted: November 15, 2003

\begin{abstract}
We have modeled the electrostatic interaction between the S4 segment of the potassium channel molecule and the surrounding water molecules on both the intracellular and extracellular sides of the neural axon cell membrane. Two methods were used to approximate this interaction: (i) a macroscopic evaluation in which the water was treated as a dielectric medium with dielectric constant 80; (ii) a microscopic evaluation considering the effects of each individual water molecule fixed in position within the water pockets surrounding the S4 segment. The potential energy of the S4 due to the water pockets was plotted against the rotation of the S4 segment, while keeping the water pockets in their fixed positions. Although the two methods gave some differing results, both methods produced single well potential energy curves of $\sim 6-9 \mathrm{eV}$ depth. Based on this energy curve, we show that other forces on the S4 must create an effective torsional spring force with spring constant $\mathrm{k} \sim 3-5 \mathrm{eV}$ in order to produce a two well potential energy curve in qualitative agreement with experimental data.
\end{abstract}

\section{INTRODUCTION}

Neurons, like the one shown in Figure 1, are made up of a cell body, a long tube-shaped axon, and a network of dendrites stemming out to thousands of other neurons. The main function of neurons is to transmit messages in the form of electrical signals that travel across each neuron, from one to the next. When this electrical signal is traveling down the length of the neural axon, it is kept moving by an important biological process of ion movement in and out of the cell.

Within the cell membrane of the neural axon are many channels, or pores, that are selectively permeable to specific molecules and ions. These channels have the ability to open and close to allow ions to move through the cell membrane into or out of the cell. For example, when a $\mathrm{K}+$ selective channel opens, $\mathrm{K}+$ ions are



Figure 1. Neuron Structure.

allowed to flow freely from the inside of the cell to the outside, and when a $\mathrm{Na}+$ channel is opened, $\mathrm{Na}+$ ions are allowed to flow from the outside in. The $\mathrm{Na}+$ and $\mathrm{K}+$ channels each play key roles in the transmission of electrical signals down the neural axon [1].

The electrical signal is both created by and the cause of movement of ions through permeable channels as the signal transmits itself downs the axon. At any given time (except when the electrical signal is being transmitted across a section of the 
cell membrane), there is a resting potential difference across the membrane. This potential difference is created by the difference in ion concentrations in the intracellular and extracellular solutions separated by the membrane. When an electrical signal is being transmitted, the potential difference across the membrane will change, causing the $\mathrm{Na}+$ channel to quickly open, allowing $\mathrm{Na}+$ ions to flow through. This movement of $\mathrm{Na}+$ ions from one side of the membrane to the other changes the concentration of ions on each side, and again changes the electric potential difference that exists across the cell membrane. The new potential difference in turn triggers the opening of the $\mathrm{K}+$ channel, which then allows $\mathrm{K}+$ ions to flow out of the cell and eventually return the potential difference across that part of the membrane back to its resting value. This process repeated over and over again along the length of the axon, creates a wave of changing potential (known as the action potential) traveling down the neuron [2]. Both $\mathrm{Na}+$ and $\mathrm{K}+$ channel operation is critical in the generation of this wave, and in all electrical signaling.

In this report, we take an in depth look at the mechanism that is involved in opening, or activating, the potassium channel of a neural axon. While experimental observations have shown a number of important facts, the complete picture of the activation process is missing. We will be examining the effect of some of the physical forces involved in activation in an attempt to add credence to experimental models, and provide insight into the fundamental principles governing the operation of potassium channels.

\section{a. The Potassium Channel}

The potassium channel is composed of a single molecule, a protein. This molecule spans the width of the cell membrane, fitting into the bilipid layer that makes up the membrane itself. The protein is composed of four repeating sections, each consisting of six a-helical transmembrane segments. These six segments are represented as long cylinders denoted S1-S6 in a schematic drawing of one segment of the channel molecule shown in Figure 2. Each of these segments is



Figure 2. Simple picture of the secondary structure of one of four homologous repeats of the potassium channel molecule [3].

connected to the adjoining ones end to end, and fits into the cell membrane, perpendicular to it. It should be noted that Figure 2 is simply a representation of the molecular structure of the protein. How the protein is positioned within the membrane is something that is yet to be fully understood. Experimental results, however, can provide an excellent guide. Figure 3 is one good educated guess of the picture of the channel molecule within the membrane.

It has been determined from experiment that the channel, the place where ions actually flow through the membrane, is formed by the S5 and S6 segments of all four sections of the channel molecule [4]. The remainder of the channel molecule, segments S1-S4, is known as the voltage sensor. It carries this name for a very simple reason. When the action potential arrives at the position of this channel, the change in potential difference is 'sensed' by the voltage sensor part of the molecule. This causes some type of conformational change in the channel molecule, causing the S5 and S6 segments to move into new positions, allowing ions to pass through the channel.

\section{b. The S4 Segment}

Experiments have shown, with certainty, that the crucial part of the voltage sensor is the S4 segment, or more precisely, the seven fixed positive charges present on the S4 segment (see Figures 2 and 3). The conformational change that is believed to cause the opening of the channel is a rotation of the S4 segment in the membrane. This result has been reached after many 




Figure 3. Hypothesized picture of two of the four repeating sections of the channel molecule in the bilipid membrane. The remaining two sections (not shown) would be present behind these two, forming a box setup with the S5 and S6 segments meeting at the center. The picture shows a suggested configuration for the closed state of a channel (after [4]).

experimental measurements involving the gating charge, which is a net movement of fixed charge across the membrane. The gating charge is best explained by a rotation of the S4 segment, but not a vertical (perpendicular to membrane) movement of the segment in the membrane [4].

A physical analysis of the rotation of the S4 segment is the main focus of our research. We will be using the model of the channel molecule shown in Figure 3 and the assumption that the rotation of the S4 is the necessary conformational change that brings about the opening of the channel. From this, we will analyze the physical interactions between the S4 segment and two key components of the S4's surroundings: the two water pockets on either side of the cell membrane (Figure 3), and the three fixed negative charges present on the S2 and S3 segments of the molecule (Figures 2, 3). We will determine the electrostatic interaction energy between these two outside forces and the seven positive S4 charges. The goal is to predict a potential energy curve that will fit, qualitatively, with the experimental results.

\section{METHODS}

There are two main forces acting on the S4 segment that we will consider: the electrostatic force of three fixed negative charges on segments S2 and S3, and the electrostatic force of the surrounding water molecules. The seven positive charges on the S4 are fixed into positions, which we will quantify shortly. It is these charges that govern the movement of the $\$ 4$ in response to the electrostatic forces. When discussing the movement and interactions of the S4 segment, we are referring only to the seven fixed charges. During the transmission of the action potential, a relatively small change in voltage of about $20-50 \mathrm{meV}$ is all that is needed to cause the potassium channel to open. The movement of sodium ions explained in the introduction causes the 20-50 meV change in potential difference. The change in energy is what acts on the seven charges to produce the conformational change (rotation) of the S4, rotating it from an inactivated position (when the ion channel is closed) to an activated position (ion channel is open and ions can flow through).

We can express this idea quantitatively using potential energy. The potential energy of the S4 segment (seven positive charges) due to the net forces acting on it must change as the S4 segment is allowed to rotate. If we were to plot potential energy versus angle of rotation for the S4, there would be two wells - one for the inactivated state and one for the activated state, since the $\mathrm{S} 4$ must be able to 
rest in both the inactivated and activated positions. The depth of these two wells could be no more than $20-50 \mathrm{meV}$, as a small change in energy must be enough to move the S4 from one well to the other. This is the result that we expect to see after considering the effects of all forces acting on the S4 segment.

\section{a. Setting Up the Problem}

Figure 4 shows the S4 segment, and the positions of the seven positive charges on the S4. These charges are arginine and lysine residues, carrying the names shown in the figure. Each residue lies on the outer surface of the S4 a-helical segment, and are about 60 degrees and 4.5 $\AA$ apart. These charges are present because of the structure of the protein, and maintain a fixed position relative to each other. Using a circular cylindrical coordinate system with its origin placed at the center of the top of the S4 cylinder, the positions of each residue are listed in table 1 , charges 0 6 . Table 1 also shows the positions of the three negative charges of the S2, S3 segments (charges 7-9) in the same coordinate system. The S4 segment is assumed to be standing perfectly perpendicular to the membrane, and stretching the entire width of the membrane, so that the top of the S4 is at the edge of the outside of the membrane, and the bottom of the S4 is at the edge of the inside of the membrane.



Figure 4. S4 segment and the relative positions of its seven positive residues.

One of the largest forces acting on the S4 turns out to be the force from the water on both the inside and outside of the cell that is in immediate contact with the S4. The first step to understanding how the water will affect the movement of the S4, is determining where the water is located in reference to the segment, and how much water is there. Using Figure 3 as a guide, we chose the shape of both the inner and outer water pockets to be a truncated half-

\begin{tabular}{|c|c|c|c|}
\hline Charge Number, $\mathbf{p}$ & Radial Position, $\mathbf{s}(\AA)$ & $\begin{array}{c}\text { Angular Position, } \\
\varphi[\text { radians] }\end{array}$ & $\begin{array}{c}\text { Vertical Position, } \\
\mathbf{Z}(\AA)\end{array}$ \\
\hline 0 & 2.5 & 0 & 0 \\
\hline 1 & 2.5 & $\pi / 3$ & -4.5 \\
\hline 2 & 2.5 & $2 \pi / 3$ & -9.0 \\
\hline 3 & 2.5 & $3 \pi / 3$ & -13.5 \\
\hline 4 & 2.5 & $4 \pi / 3$ & -18.0 \\
\hline 5 & 2.5 & $5 \pi / 3$ & -22.5 \\
\hline 6 & 2.5 & $6 \pi / 3$ & -27.0 \\
\hline 7 & 6.5 & 0 & -13.5 \\
\hline 8 & 5.5 & $9 \pi / 6$ & -18.0 \\
\hline 9 & 7.5 & $5 \pi / 3$ & -22.5 \\
\hline 10 & 10.0 & $2 \pi / 3$ & -25.0 \\
\hline
\end{tabular}

Table 1. The positions of the positive residues (Figure 4) and four other negative charges (7-10) on nearby $\alpha$-helical segments. These values agree with those of Chancey and George [5]. 


\begin{tabular}{|c|c|c|c|}
\hline \multicolumn{4}{|c|}{ Dimensions of Water Pockets } \\
\hline \multirow{4}{*}{ Inner } & $\mathrm{s}$ & $\varphi$ & $\mathrm{Z}$ \\
& $\begin{array}{c}\text { Upper Base: } \\
5 \AA\end{array}$ & $3 \pi / 12$ & $-35 \AA$ \\
& $\begin{array}{c}\text { Lower Base: } \\
15 \AA\end{array}$ & $13 \pi / 12$ & $-4 \AA$ \\
Outer & $\begin{array}{c}\text { Upper Base: } \\
15 \AA\end{array}$ & $15 \pi / 12$ & $-14 \AA$ \\
& $\begin{array}{c}\text { Lower Base: } \\
5 \AA\end{array}$ & $\pi / 12$ & $5 \AA$ \\
\end{tabular}

cone with the center of the S4 segment at the center of the cone. Figure 5 provides a picture of the shape of the inner membrane water pocket. The outer membrane pocket would look like a flipped version of the inner pocket, on the opposite side of the S4 segment. The dimensions of both water pockets according to the same coordinate system used for the S4 are also listed in Figure 5. The absolute $\varphi$ dimensions for the water pockets are really arbitrary, since we will eventually be rotating the $S 4$ segment through the entire $2 \pi$ radians.

It should be noted that the aqueous solution on both the inside and outside of the cell membrane is not just water, but is a saline solution containing different concentrations of several different ions, such as potassium, sodium, calcium, and chlorine. A proper analysis of the effects of the 'water' surrounding the S4 would include the effects of the ions present in the saline solution. However, due to the relatively small size of both water pockets, the ions present in the solution are not of great concern. Using the macroscopic concentrations of ions in cellular solutions [6], we have calculated a (0.70) probability of a single positive ion being in the inner water pocket as we defined it, and a (0.40) probability of a negative ion being in the outer pocket. To include the effect that these ions would have, we chose to place a single positive ion at a fixed position toward

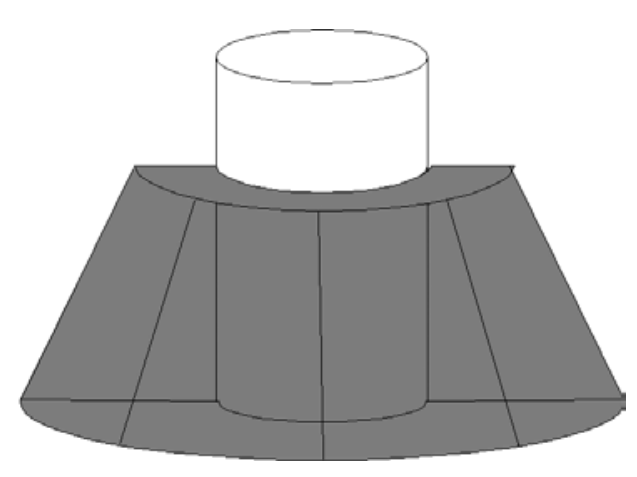

Table 2. (At left) lists the dimensions of the inner and outer water pockets adjacent to the S4 segment.

Figure 5. (Above) illustrates the geometry of the inner pocket.

the outside of the inner water pocket (position given in table 1, charge 10), and to ignore the effect of an ion that might be in the outer water pocket [7].

As mentioned before, we are concerned with finding the potential energy of the S4 segment due to the external forces acting on it. The force that the water pockets exert on the S4 is an electrostatic force. The seven positive charges on the S4 will cause the water around them to be slightly polarized, meaning that the negative ends of the water molecules will be attracted to the seven positive residues. This attraction will result in a negative potential energy, and the S4 will want to move to a position where the charges will be more immersed in water than anywhere else. We can find the potential energy by finding the potential at the position of each S4 residue due to the polarized water. Multiplying this times the charge (e) at each position and adding gives the total potential energy of the S4 due to the water. So, the first step then becomes determining the polarization of the water, i.e. how the water molecules are positioned in relation to the S4 charges. The positioning of the water can be determined from the total electric field within the water pocket. We examined this problem in two different ways: (i) a macroscopic evaluation treating water as a continuous dielectric medium, and (ii) a microscopic evaluation considering the 
effects of each individual water molecule fixed at a given location within the water pockets.

\section{b. Polarization Model}

In this method, we attempt to use macroscopic electrostatics principles to explain the interaction between the water and the S4 residues. We treated the water in each of the pockets as a linear dielectric medium with a dielectric constant of $\mathrm{k}=80$. For a linear dielectric, the polarization of the dielectric is proportional to the total electric field:

$$
P=\chi_{e} \varepsilon_{0} E
$$

where $\chi_{e}=\kappa-1$

is the electric susceptibility, and $\varepsilon_{0}$ is the permittivity of free space. $\mathrm{E}$, the total electric field in the water pockets will be a function of the charges that contribute to the electric field. This total electric field is dominated by the 11 fixed point charges (7 S4 residues and 3 negative residues fixed on the channel molecule, and the positive ion in the inner water pocket that was assigned a fixed position) given in table 1 . Although other factors contribute to the electric field, these eleven charges will be the main contributors.

Throughout this report, we ignore any effect that the bulk of the channel molecule protein has on the total electric field within the water pockets. Although some proteins have been found to have dielectric properties $(\kappa \sim 2-5)$ on the macroscopic level, it is unclear how the channel molecule would react on such a small scale, and reacting to charges within the protein itself. For simplicity, we have treated the entire protein, except for the ten charged residues, as empty space.

The electric field created by the 11 fixed charges is found from the potential

$$
V(r)=\sum_{i=1}^{11} \frac{q}{4 \pi \varepsilon_{0} d_{i}}
$$

where $d_{i}$ is the distance from the $i^{\text {th }}$ residue to the field point $r$, and $q$ is either $+e$ or $-e$. The electric field is then

$$
\vec{E}=-\vec{\nabla} V
$$

We now know the electric field at every point in the dielectric due to the external charges. From the definition of linear dielectrics, the total electric field inside the dielectric is just $1 / \mathrm{K}$ times the external electric field (Applying this simple method to a non-symmetric dielectric is an approximation, which we will address in more detail shortly). From the total electric field, we are also given the polarization by equation (1). Due to the large value of the electric field within a few angstroms of a point charge, a portion of each water pocket (the dielectric) will be saturated. We will represent this saturation by placing an upper value on the polarization of water at $0.209 \mathrm{C} \cdot \mathrm{m}$ [6].

From the polarization, we can find the potential at every point in space created by the polarized water:

$$
V(r)=\frac{1}{4 \pi \varepsilon_{0}} \int_{V} \frac{\vec{d} \cdot \vec{P}\left(\overrightarrow{r^{\prime}}\right)}{d^{2}} d r^{\prime 3}
$$

where $d$ is the distance from the distance from the residue to the integration point r', and $P\left(r^{\prime}\right)$ is the polarization at the integration point $r^{\prime}$ [8]. Remember that the effect of the water on $V$ is contained in the $P\left(r^{\prime}\right)$ term. The integral was evaluated numerically at the position of each of the seven S4 residues to give the potential, and consequently the potential energy, of each of the seven S4 residues, due to the water. The total potential energy of the S4 is just the sum of the values from the seven individual residues. This same total value was calculated for different angles of rotation of the S4 segment.

Keeping the water pockets in fixed positions, the S4 segment was allowed to rotate, changing the angular positions of all of the charges. The angular positions of the charges were the only thing that was changed. The position of the charges on the s- and z-axes remained fixed. As the charges changed positions, the magnitude of the potential energy between the charges and the water changed. A graph of energy versus angle of rotation (with radian positions defined by Figure 4 and table 1) was plotted giving a potential energy curve. This curve is the water's contribution to the 
total two-well potential energy curve that we will have after adding the potential energy curves of all important factors.

As mentioned before, this method is just an approximation, because of some flaws with the mathematical procedure. Allowing the polarization of the water to be exactly proportional to the total electric field is a correct assessment, but the 'total' electric field at every point will be different than what we assigned it to be. Taking $1 / \mathrm{k}$ times the external electric field only works for a completely symmetric dielectric medium. For odd-shaped dielectric mediums like our water pockets, this will not be true. The difference comes from the internal electric field created by the dielectric. For example, in the case of a sphere all non-radial parts of the electric field created by a small section of the dielectric is canceled by the fields of the surrounding sections. Without symmetry, this canceling effect is limited, and the total electric field becomes dependent on the shape of the dielectric medium. We are still unsure of exactly how much our calculations are affected by using this approximation.

\section{c. Individual Dipole Method}

We are unsure how effective it is to treat such a small area of water using macroscopic principles, and keeping in mind some of the problems with the polarization model, we decided to do a more microscopic evaluation as an alternative. For this model, we looked at each water molecule individually, treating them as perfect dipoles, and assigning each a fixed position in space. The water pocket was now composed of about $100-200$ individual water dipoles, rather than a continuous dielectric medium. To find the potential energy, we calculated the field produced by each individual dipole, and the energy of the S4 residues within that field.

The positioning of the water molecules around the S4 was done in a simple, block arrangement. Using the density of water as $1 \mathrm{~g} / \mathrm{mL}$, we calculated that a single water molecule takes up, on average, $3.1 \AA^{3}$ of space. Keeping the circular cylindrical coordinate system from before, we assigned positions to each water molecule in a block style, spacing them 3.1 $\AA$ apart in each dimension, and placing them out as far as the dimensions of our water pockets.

While we want to find the potential energy of the seven S4 residues due to the water molecules, an equivalent statement would be to find the potential energy of all the water molecules due to the seven positive S4 charges. Since the latter proved to be a simpler calculation, that method was used. The equation for the energy of a dipole in an electric field is

$$
U=-\vec{p} \cdot \vec{E}
$$

The electric field in this equation for any given dipole is just the electric field of the seven S4 residues at the position of that dipole. All that we need to find now is the direction and magnitude of each of the individual water dipoles. The water dipoles will tend to line up in the direction of the total electric field within the water pocket. Similarly to before, the total electric field inside the water pocket is dominated by the eleven fixed charges (table 1). We used the electric field of the eleven fixed charges to set the direction of the dipoles in place.

The magnitude of $p$ for each dipole is the magnitude of the dipole moment of a water molecule $\left(6.237 \times 10^{-30} \mathrm{C} \cdot \mathrm{m}\right)$ with a correction. The actual magnitude of $p$ in the above formula will not be exactly equal to the magnitude of the dipole moment of water, because the dipoles are never lined up completely with the field. Thermal energy will have each molecule (dipole) fluctuating in direction, but with an overall direction (over time) that is lined up with the total electric field. The magnitude depends on the probability that the dipole will be in the energy state that has it lined up with the field. We calculated the probability of the dipole being lined up with the electric field based on the strength of the electric field using [9]

$$
P=\frac{e^{(-\mathrm{E} / k T)}}{\int n(\mathrm{E}) e^{(-\mathrm{E} / k T)} d \mathrm{E}}
$$

where $\mathrm{k}$ is Boltzman's constant, $\mathrm{T}$ is the absolute temperature in degrees Kelvin, E is the energy of a state (equal to $-\vec{p} \cdot \vec{E}$ ), and $n(E)$ is the number of states of energy E. 
We multiplied this probability times the dipole moment of water to get the magnitude of each water dipole. Completing the energy formula, the potential energy of each dipole in the field of the seven S4 residues can now be found. Using a numerical program like the one we used in the polarization model, we found the potential energy of the S4 due to all water dipoles together at different angles of rotation, and plotted a potential energy curve from the data.

The major difference between this model and the polarization model is the use of individual dipoles rather than a continuous dielectric, but the problem of the total electric field still exists. Once again, the water itself (this time, the dipoles) will have an effect on each other such that the total electric field at a point inside the water pocket is something different from the field due to the eleven fixed charges. To attempt to compensate for this, we calculated the electric field produced by each of the water dipoles, and allowed these to influence the total electric field inside the pocket. Since we cannot know the electric field produced by a water dipole until we know which direction the dipole is pointing, we began the calculation having the total electric field coming from just the eleven fixed charges. After calculating the positions of the dipoles from this field, we added in the electric field of all dipoles to produce and update the total electric field and recalculated the positioning of the dipoles. Again, we took the new dipole directions and recalculated the field, and so on, allowing the process to repeat for several iterations. The idea was to continue to do this until equilibrium was reached where the dipoles would be pointing in the correct direction. While the energy values at each step did begin to converge, they did not converge to one number. At the end of several iterations, we took the average of the last two energy values, which were always reasonably close to each other. A more complete answer would need to include a mean field theory analysis to get the correct directions of all the water molecules.

\section{d. Negative Residues and Ion}

In both of the models, we considered the effects of the three negative charges and single positive ion in the determination of the electric field inside the water pocket, but did not consider the direct effects of the four charges on the S4 segment. Each of these charges will have either an attractive or repulsive force acting on the seven S4 residues that needs to be accounted for. The calculation for this is simple, since all we are dealing with is point charges. The potential at the position of each of the S4 residues due to the four other fixed charges was determined, and the potential energy taken from this. (In this calculation, $\varepsilon_{0}$ was used instead of an $\varepsilon$ value that takes account for the water dielectric, because we have already considered the effects of the water molecules. Because we allowed the water molecules to react to the four other fixed charges when we determined the energy for the water pocket, we need not introduce the water again here.) The energy is then a simple point charge in space calculation. Once again, the energy was plotted against the angle of rotation of the S4, as the four external charges do not change position even while the S4 is rotating. The resulting curve was added to the potential energy curves from each of the two models, to get a total interaction energy of water, negative residues, and ion for both the polarization and individual dipole models.

\section{RESULTS}

Figures $6 a$ and $6 \mathrm{~b}$ show the graphs of potential energy for both the polarization $(P)$ and the individual dipole (ID) models before the addition of the four external charges. The graphs both show a sinusoidal shaped curve that has one maximum and one minimum at relatively similar positions (minimum at about $4-4.5$ radians). Comparing the graphs, we see that the absolute scale of the polarization curve is larger than that of the individual dipole curve, but the relative scale of both curves is very comparable. Figure 6e shows the graph of the energy from the four external charges. Figures $6 c$ and $6 d$ show the final energy curves for $P$ and ID models, including a best-fit sine curve approximating the data curve.

The ID graphs appear choppy, which is a result of the setup of the water molecule positioning in the individual dipole 


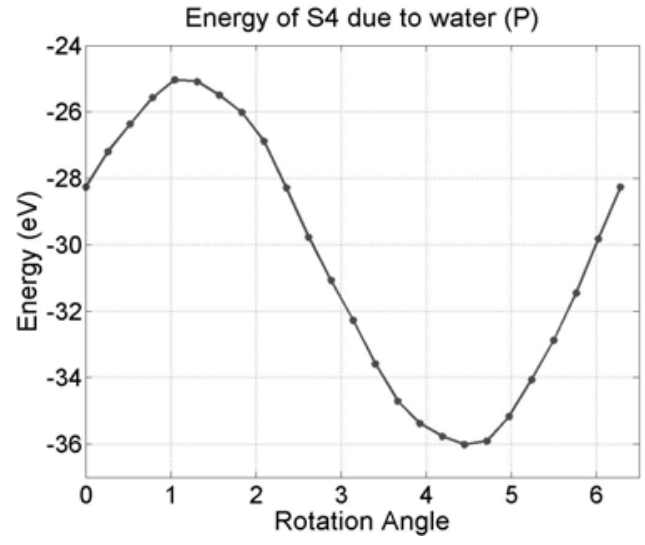

(a)

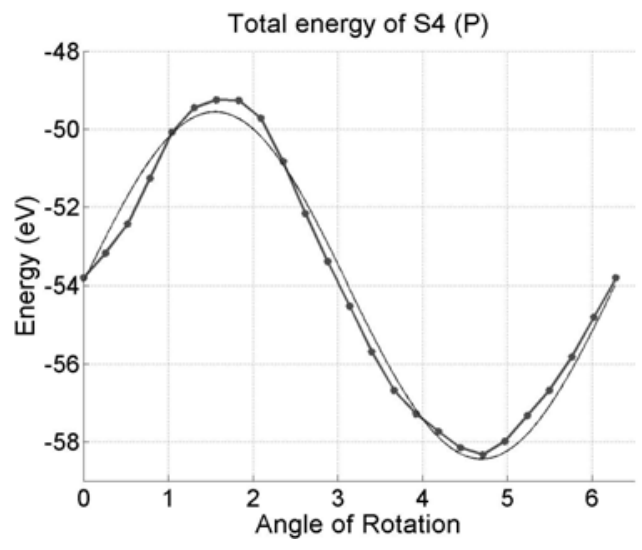

(c)

Figure 6. Graphs of potential energy using the polarization method (a) and the individual dipole method (b). Curves (c) and (d) show the total potential energy obtained by adding the effects of the four external negative charges-where, as before, the polarization method is at left (c) and the individual dipole method is at right (d). Graph (e) shows the potential energy curve that arises due to just the four external negative charges. All five graphs (a-e) have vertical axes in eV; all horizontal axes are in radians for the angle that the $S 4$ segment rotates through.

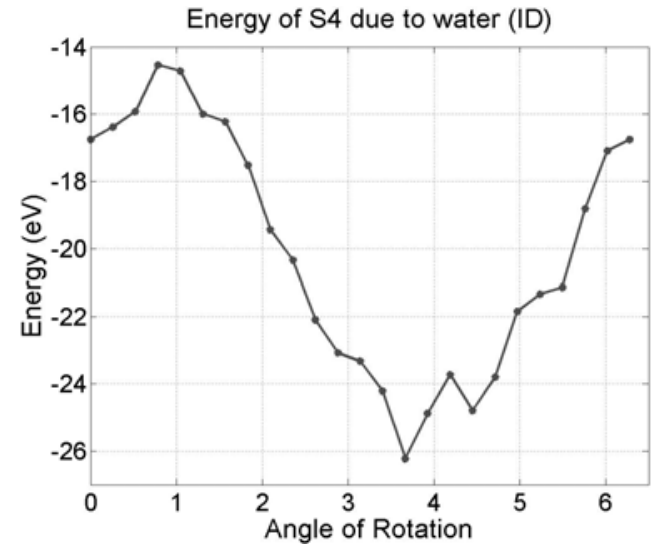

(b)



(d)

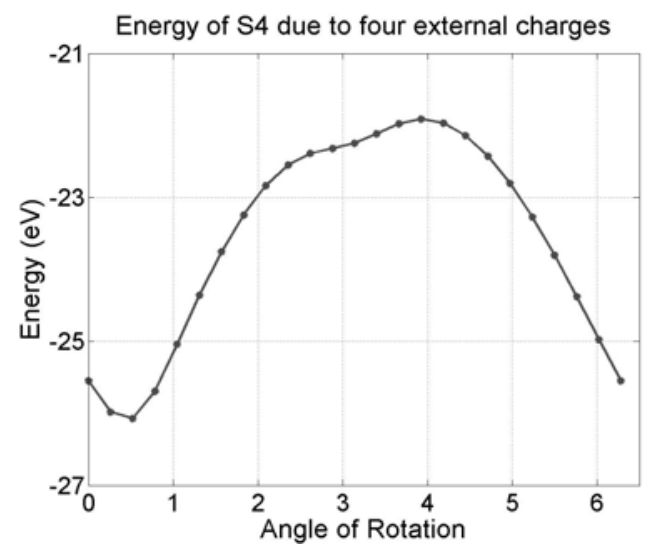

(e) 
model. The block setup that we used fills up the water pocket completely with water. The problem is with the water molecules close to the S4 residues. In the computational analysis, we set the radius of each of the $\mathrm{S} 4$ residues to be $1.5 \AA$, so each of the residues will be 'sticking out' $1.5 \AA$ from the edge of the S4 segment. Since a water molecule and a S4 residue can not both occupy the same space, the water molecule in question was simply removed whenever it was too close to the positions of the residues. As we allow the S4 to rotate, the residues move, and different water molecule positions are taken away each time. This is enough of a factor to cause the choppiness in the graphs in Figure 6 , but the overall shape is maintained.

\section{CONCLUSIONS}

The potential energy minimum at $\sim 4$ radians tells us that the water solution (water + ion) and fixed negative charges will push the S4 towards the higher radians, which has (the way we set our coordinates up) commonly been thought to be closer to the activated state than the inactivated one. Also, since we only have a single well when we expected to see a double well, there is clearly more forces contributing to the actual potential energy than we have considered. Combining this with the relative depth of both wells at $\sim 7-8 \mathrm{eV}$, which is much larger than our original expectations, leads us to draw one important conclusion about other forces acting on the S4.

\section{a. Introducing a Torsional Spring Force}

From the simple fact that potassium channel activation happens, we know that there must be a two-well potential energy with well depths of $\sim 20-50 \mathrm{meV}$. Since the electrostatic interactions we have considered give us a result far from this twowell curve, the rest of other forces acting on the S4 must create a potential energy function that, when added to ours, produces the necessary two-well function. (If it does not, than there is either a problem with our calculations, or a problem with this particular model of the potassium channel.) We have combined the remaining forces into an effective torsional spring energy:



(a)

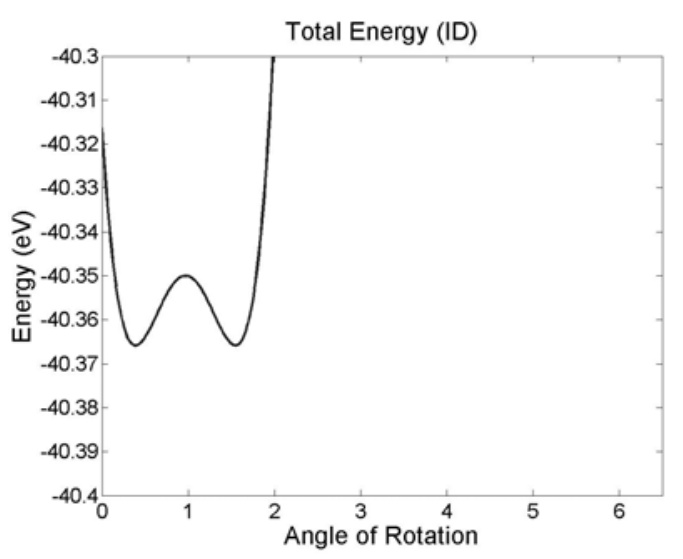

(b)

Figure 7. Two graphs of total potential energy, including the effect of a torsional spring restoring term. Graph (a) follows from the polarization method and uses a spring constant $\mathrm{k}=4.2 \mathrm{eV}$ and an angular offset of $\theta_{0}=1.6$ radians. Graph (b) follows from the individual dipole method, with a $\mathrm{k}=$ $3.3 \mathrm{eV}$ and $\theta_{0}=1.0$ radians.

$$
U_{t o r}=\frac{1}{2} k\left(\theta-\theta_{0}\right)^{2}
$$

There must be some value of $k$ and $\theta_{0}$ that will give a two-well potential energy curve when we add $U_{\text {tor }}$ to our calculated graphs.

We carried out this step simply by adding up our best-fit sine curves for each model to the torsional energy curve at different values of $k$ and $\theta_{0}$, until we found the values that produced the correct energy 
curve. The resulting energy curves for each model are shown in Figure 7. The polarization model gave a value of $\mathrm{k} \sim 4 \mathrm{eV}$ and $\theta_{0} \sim 1.5$ radians, and the individual dipole model gave values of $\mathrm{k} \sim 3 \mathrm{eV}$ and $\theta_{0}$ $\sim 1.0$ radians.

These numbers give us an order-ofmagnitude estimate of the impact that some other forces must have on the S4 for the model in Figure 3 to hold true. Most likely, this force will be created by the structure of the molecule. The backbone structure of the protein will resist any rotation of the S4 segment, and strains will be placed on the S4 by the adjoining segments to push it back to the resting position $\left(\theta_{0}\right)$ in the molecule structure. Improvements in the numerical methods used, and more attention to detail could improve upon the accuracy of the calculations shown in this report, but the results will still produce a similar potential energy curve, and values of $k$ and $\theta_{0}$ within an order of magnitude of the values listed above.

\section{ACKNOWLEDGEMENTS}

I would like to thank Dr. C. C. Chancey for his guidance and support, and the University of Northern lowa for providing me with an undergraduate summer research fellowship.

\section{REFERENCES}

1. Gary Yellen, The Voltage-Gated Potassium Channel and Their Relatives. Nature, vol. 419 (5 September 2002) pp. 35-42.
2. Bertil Hille, Ionic Channels of Excitable Membranes. (Sinauer Associates Inc., Sunderland, MA, 1992).

3. A. Cha, G. Snyder, P. R. Selvin, and F. Bezanilla, Atomic scale movement of the voltage-sensing region in a potassium channel measured via spectroscopy. Nature. Vol. 402 (16 December 1999) pp. 809-813.

4. F. Bezanilla, The Voltage Sensor in Voltage-Dependent Ion Channels, Physiological Reviews. Vol. 80 no. 2. (April 2000) pp. 555-592.

5. C. C. Chancey and S. A. George, Physical Model of Voltage Sensing in Sodium Channels Based on the Sliding Helix Complex. Physical Review E. vol. 53 (May 1996) pp. 5137-5145.

6. Russell K. Hobbie, Intermediate Physics for Medicine and Biology (Springer, New York, 1997).

7. Leon D. Islas and Fred J. Sigworth, Electrostatics and the Gating Pore of Shaker Potassium Channels. J. Gen. Physiol, vol. 117 (January 2001) pp. 6989.

8. David J. Griffiths, Introduction to Electrodynamics. (Prentice Hall, Upper Saddle River, NJ, 1999).

9. Charles Kittel and Herbert Kroemer, Thermal Physics (Freeman, New York, 1980).

University of Northern Iowa : Cedar Falls, Iowa USA

